\title{
Free Inter-band Aliasing Sub-band Adaptive Filtering with Critical Sampling Filter bank Analysis
}

\author{
Prabhakar.Telagarapu \\ GMRIT, Rajam \\ Srikakulam Dist \\ A.P, 532127, India
}

\author{
Dr.K.Satya Prasad \\ Professor (ECE) \\ University College of Engineering \\ JNTU Kakinada, A.P, India
}

\author{
P.M.K.Prasad \\ GMRIT, Rajam \\ Srikakulam Dist, \\ A.P, 532127, India
}

\begin{abstract}
Adaptive Filtering is an important concept in the field of signal processing and has numerous applications in fields such as speech processing and communications. Examples in speech processing include speech enhancement, echo and interference cancellation and speech coding. An Adaptive filter is a filter that self-adjusts its transfer function according to an optimizing algorithm. Because of the complexity of the optimizing algorithms, most adaptive filters are digital filters that perform digital signal processing and adapt their performance based on the input signal. An Adaptive filter is often employed in an environment of unknown Statistics for various purposes such as system identification, inverse modeling for channel equalization, adaptive prediction and interference canceling. Knowing nothing about the environment, the filter is initially set to an arbitrary condition and updated in a step by step manner towards an optimum filter setting. For updating, the least meansquare algorithm is often used for its simplicity and robust performance. However, the L MS algorithm exhibits slow convergence when used with an ill-conditioned input such as speech and requires a high computational cost, especially when the system to identified has a long impulse response. Simulations show that the proposed structure converges faster than both an equivalent full band structure at lower computational complexity and recently proposed SAF structures for a colored input. The analysis is done using MATLAB, a language of technical computing, widely used in Research, Engineering and Scientific computations.
\end{abstract}

Index Terms: Adaptive filtering, aliasing, Critical Sampling, LMS Algorithm.QMF.

\section{INTRODUCTION}

An adaptive filter is often employed in an environment of unknown statistics for various purposes such as system identification, inverse modeling for channel equalization, adaptive prediction, and interference cancelling. Knowing nothing about the environment, the filter is initially set to an arbitrary condition and updated in a step-by-step manner toward an optimum filter setting. For updating, the least mean square (LMS) algorithm is often used for its simplicity and robust performance. However, the LMS algorithm exhibits slow convergence when used with an ill-conditioned input such as speech and requires a high computational cost, especially when the system to be identified has a long impulse response .One promising method that improves the performance and reduces the computational cost is sub band adaptive filtering (SAF), in which the input is decomposed into a number of sub band signals, and the adaptive filtering is performed on each sub band. It has the potential for a faster convergence and a lower computational complexity than a full band structure. However, a sub band structure suffers from two deficiencies. First, the inter band aliasing that is introduced by the down sampling process required in reducing the data rate is unavoidable and degrades the performance. Second, the filter bank introduces additional computation and system delay. For these reasons, various SAF structures were proposed. In this project, a critically sampled SAF structure that is almost alias-free is proposed to reap all the benefits of using an SAF. Since the proposed SAF is performed using sub bands that are almost alias-free, there is little inter band aliasing error at the output. In each sub band, the inter band aliasing is obtained using a bandwidth-increased linear-phase FIR analysis filter, whose pass band has almost-unit magnitude response in the sub band interval, and is then subtracted from the sub band signal. This aliasing cancellation procedure, however, causes the spectral dips of the sub band signals. These spectral dips can be reduced by using a simple FIR filter.

\section{FULL BAND ADAPTIVE FILTER}

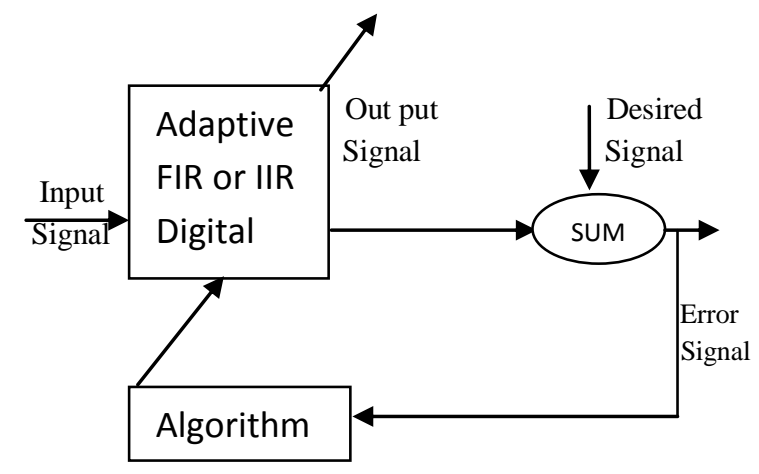

Fig. 1.Full band Adaptive Filtering

Figure shows a block diagram in which a sample from a digital input signal $x(k)$ is fed into a device, called an adaptive filter, that computes a corresponding output signal sample $y(k)$ at time $\mathrm{k}$. For the moment, the structure of the adaptive filter is not important, except for the fact that it contains adjustable parameters whose values affect how $\mathrm{y}(\mathrm{k})$ is computed. The output signal is compared to a second signal $\mathrm{d}(\mathrm{k})$, called the desired response signal, by subtracting the two samples at time $n$. This difference signal, given by $e(k)=d(k)-y(k)$ is known as the error signal. The error signal is fed into a procedure which alters or adapts the parameters of the filter from time $\mathrm{k}$ to time $\mathrm{k}+1$ in a well-defined manner. This process of adaptation is represented by the oblique arrow that pierces the adaptive filter block in the figure. As the time index $\mathrm{k}$ is incremented, it is hoped that the output of the adaptive filter becomes a better and better match to the desired response signal through this adaptation process, such that the magnitude of $e(k)$ decreases over time. 
In this context, what is meant by "better" is specified by the form of the adaptive algorithm used to adjust the parameters of the adaptive filter. In the adaptive filtering task, adaptation refers to the method by which the parameters of the system are changed from time index $\mathrm{k}$ to time index $\mathrm{k}+1$. The number and types of parameters within this system depend on the computational structure chosen for the system. So An adaptive filter designs itself based on the characteristics of the input signal to the filter and a signal that represents the desired behaviour of the filter on its input. Designing the filter does not require any other frequency response information or specification. To define the self-learning process the filter uses, you select the adaptive algorithm used to reduce the error between the output signal $y(k)$ and the desired signal $d(k)$.When the LMS performance criterion for $e(k)$ has achieved its minimum value through the iterations of the adapting algorithm, the adaptive filter is finished and its coefficients have converged to a solution. Now the output from the adaptive filter matches closely the desired signal $d(k)$. When we change the input data characteristics, sometimes called the filter environment, the filter adapts to the new environment by generating a new set of coefficients for the new data. Notice that when $e(k)$ goes to zero and remains there you achieve perfect adaptation, the ideal result but not likely in the real world.

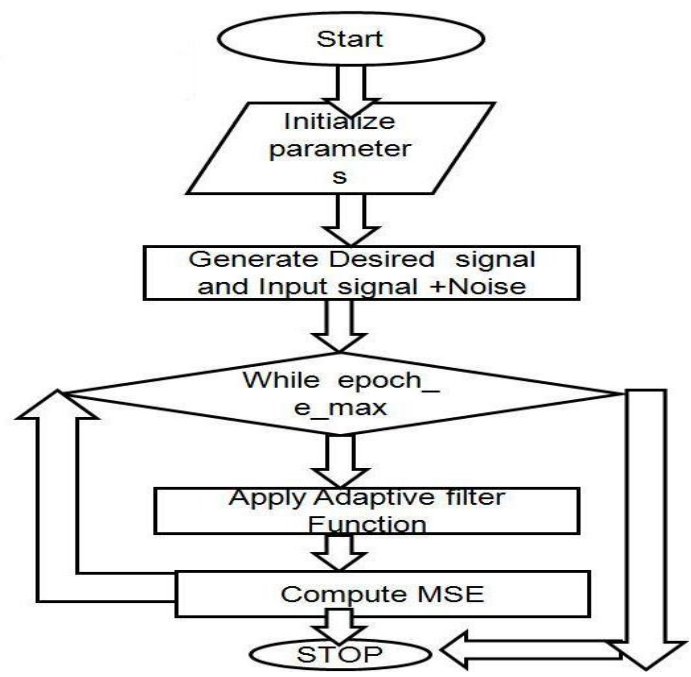

Fig. 2.Flow Chart of Full band Adaptive Filtering

Filter Input(Signal+Noise)
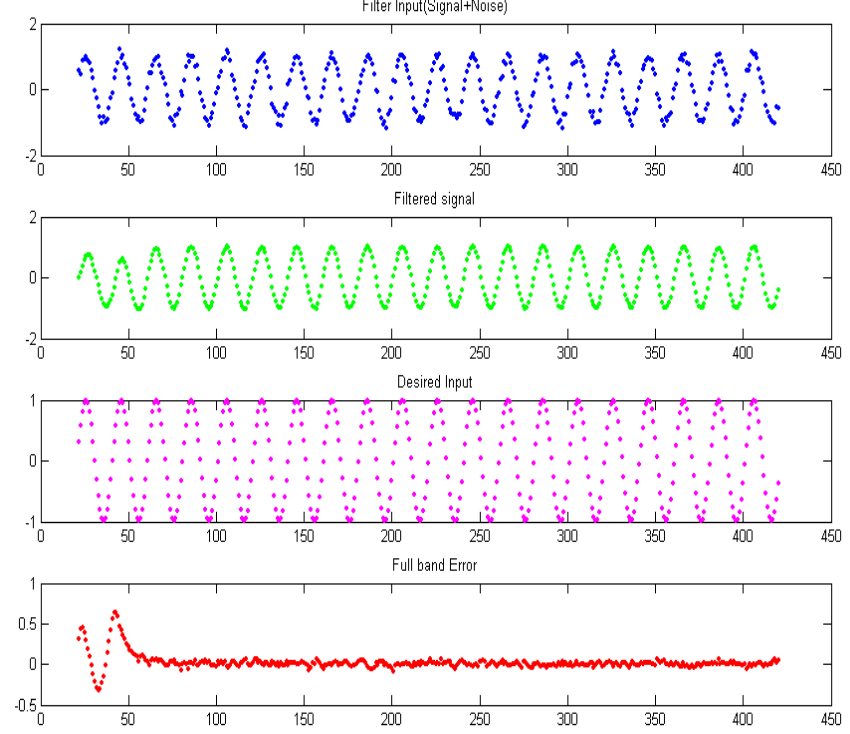

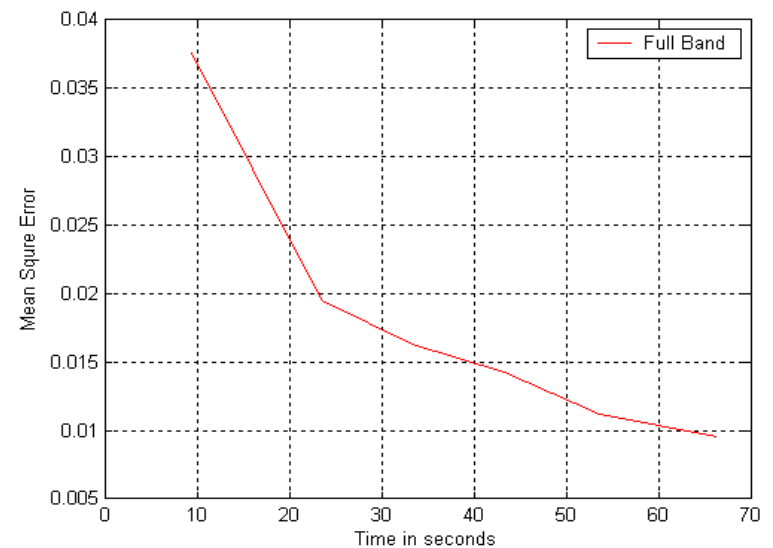

Fig. 3.Simulation Results of Full band Adaptive Filtering

Above fig shows that the fullband convergence behavior is incresed when iterations increased. Here we plot the diagram between Time and Mean square error(MSE).

\section{3 .SUB BAND ADAPTIVE FILTERING}

In SAF, signals are decomposed into a number of sub band signals using an analysis filter bank, and the adaptive filtering is performed on each sub band. The result in each sub band is combined into an output using a synthesis filter bank. If the sub band signals are band limited to frequency ranges much smaller than that of the original input signal they can be down sampled before processing. Because of the lower sampling rate, the processing of the down sampled signals can be carried out more efficiently. After processing, these signals are up sampled before being combined by the synthesis bank into a higher rate signal. The combined structure employed is called a quadrature mirror filter (QMF) bank. If the down sampling and up sampling factors are equal to or greater than the number of bands of the filter bank, then the output can be made to retain some or all of the characteristics of the input by properly choosing the filters in the structure. In the case of equality, the filter bank is said to be a critically sampled filter bank. The most common application of this scheme is in the efficient coding of a signal. Block diagram of the adaptive filtering in the kth subband. $X(z), H_{k}(z), G_{k}(z)$ and $E_{k}(z)$ represent the $z$-transforms of input, the kth analysis filter, the kth adaptive filter, and the kth error signal for $\mathrm{k}=0,1, \ldots, \mathrm{M}-1$, respectively.In SAF, signals are decomposed into a number of subband signals using an analysis filter bank, and the adaptive filtering is performed on each subband. The result in each subband is combined into an output using a synthesis filter bank. For a critically sampled SAF with M subbands, the kth subband error $\mathrm{E}_{\mathrm{k}}(\mathrm{z})$, shown in Fig., 


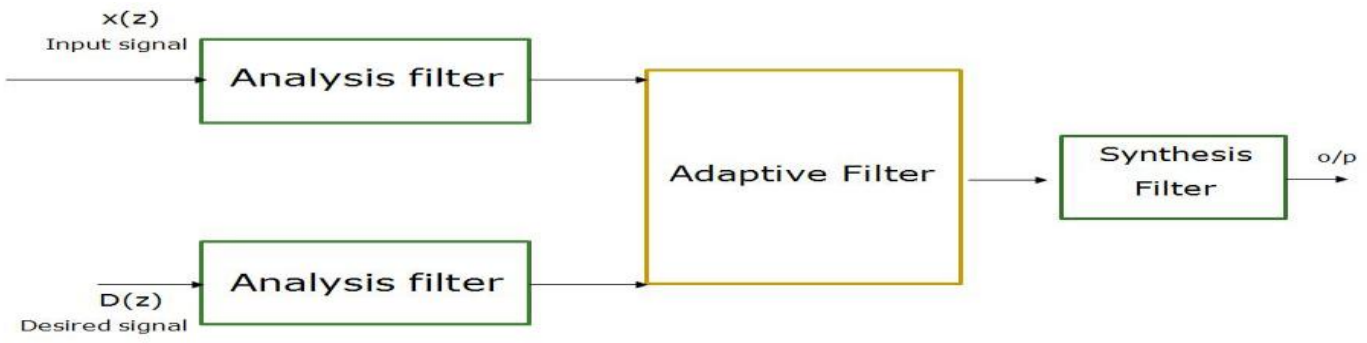

Fig. 4.Subband Adaptive Filtering

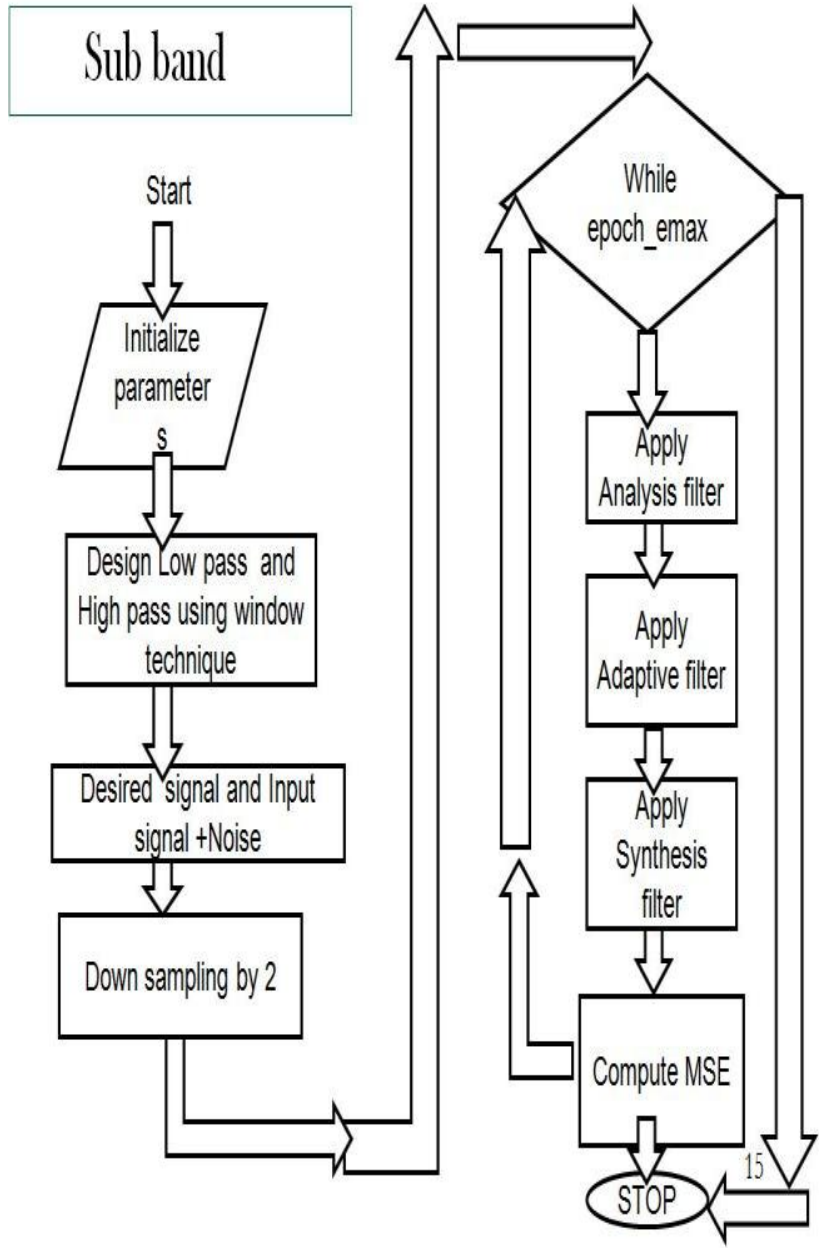

Fig. 5 . Flow Chart of Sub-band Adaptive Filtering

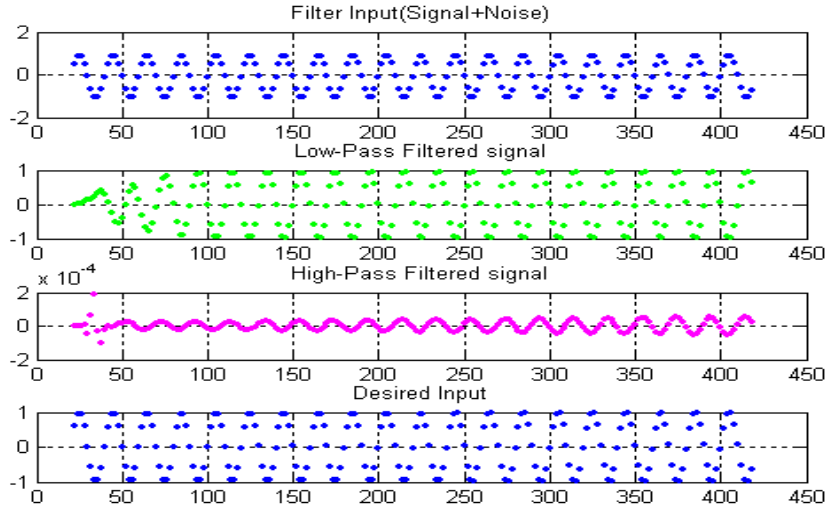

Fig. 6 .Simulation Results Of Subband Adaptive Filtering

\section{ALIAS FREE SUB BAND ADAPTIVE FILTERING}

The inter band aliasing is a major bottleneck in using SAF, and several methods for reducing the inter band aliasing have been proposed. In this paper, critically sampled SAF that is almost alias-free is proposed. The inter band aliasing components are caused by down sampling the signal which has passed through a non ideal analysis filter: the down sampling process is essential in almost all multi rate signal processing for making the overall data rate nearly equivalent to that of the input. Fig .shows the magnitude responses. The inter band aliasing is extracted in each sub band using the bandwidth-increased FIR linear-phase analysis filters and then subtracted from each sub band signal. The almost alias-free sub band signals have spectral dips, so the spectral dips are reduced using a filter for the spectral flatness and then the outputs are used for adaptive filtering in each sub band. The computational complexity of the proposed SAF algorithm is approximately reduced compared to that of the full band algorithm. Simulations results show that the proposed sub band structure achieves better convergence rate than the full band structure for random noise input at lower computational complexity.

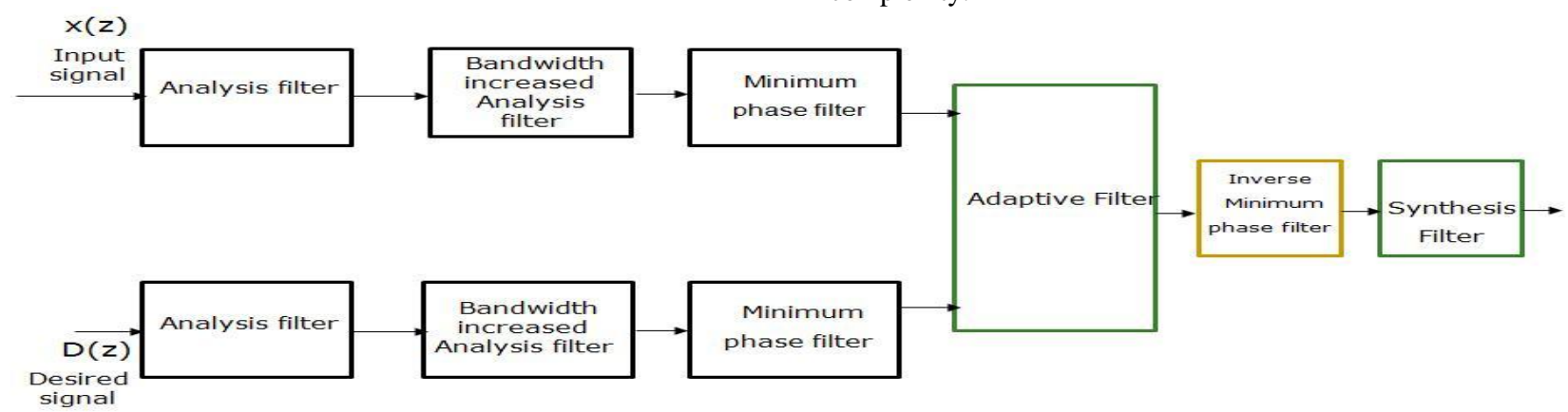

Fig. 7 . Free Interband Aliasing SAF 

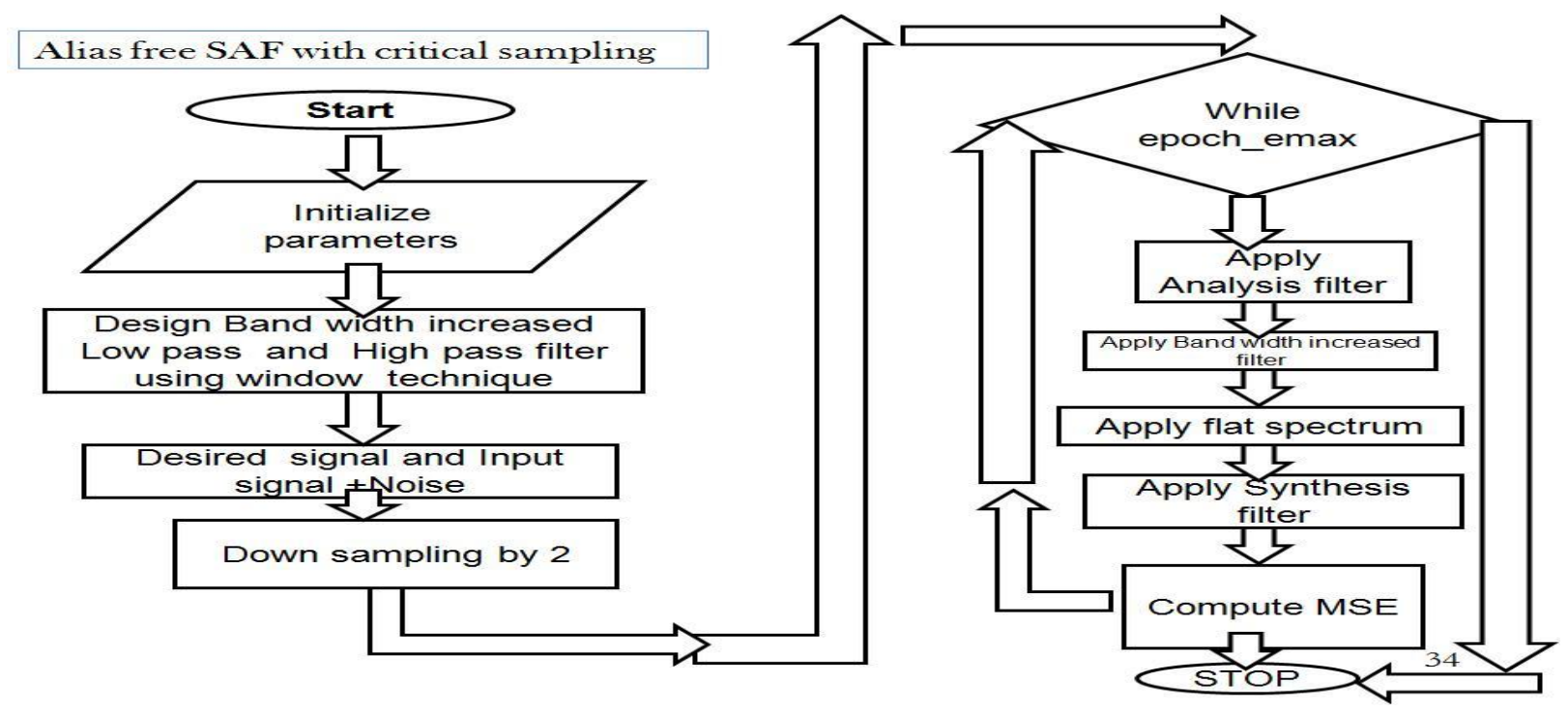

Fig. 8.Flow Chart of Free Interband Aliasing SAF
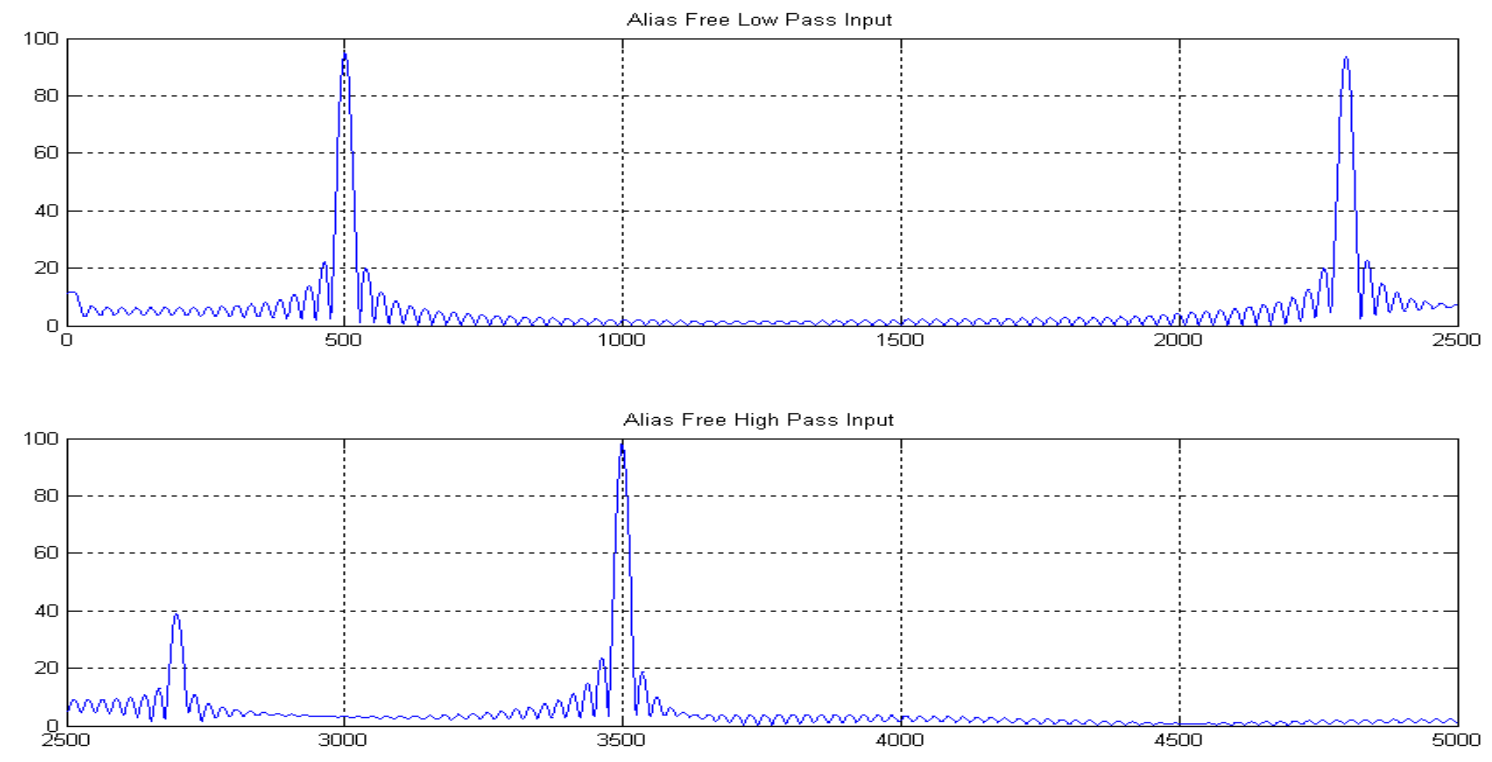

Fig.9 Extraction of free Interband Aliasing LP and HP

Figure shows Alias free low pass and high pass adaptive filter input, here extracted signals are $500 \mathrm{~Hz}, 2300 \mathrm{~Hz}, 2700 \mathrm{~Hz}$ and $3500 \mathrm{~Hz}$.

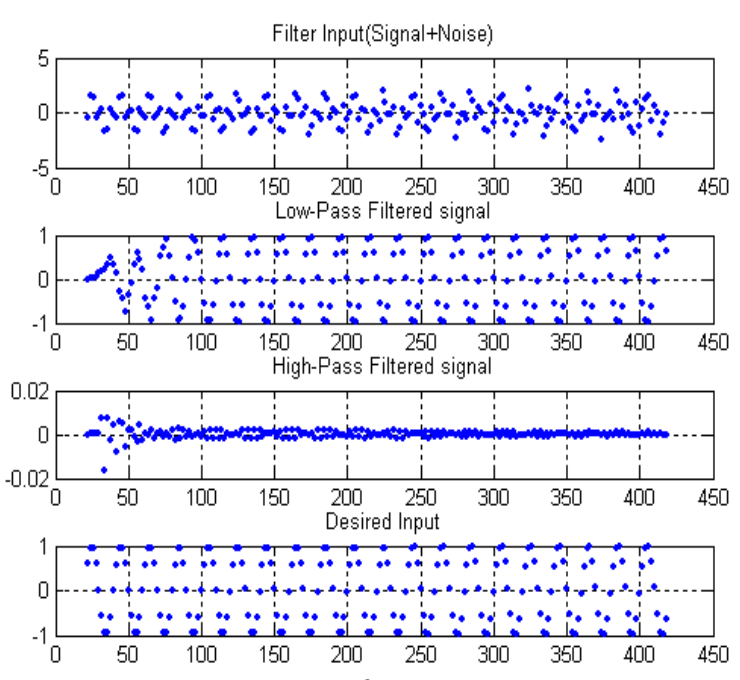

Fig. 10.Simulation Results of Free Interband Aliasing SAF

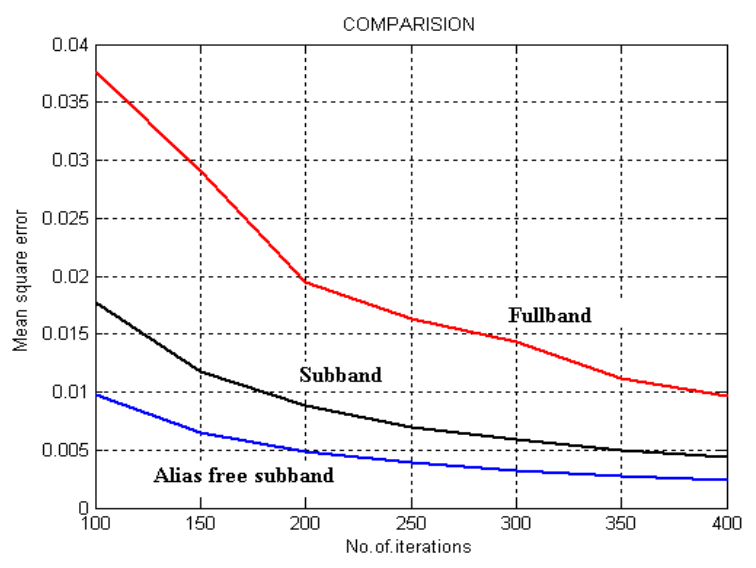

Fig. 11 Error Comparision

While comparing Alias free subband adaptive filter error performance is very less than subband adaptive filter and full band adaptive filter. Here observed that fullband, Subband and 
Alias free subband adaptive filter error is decreases while increasing the iterations. Here maximum no.of iterations 400 used.

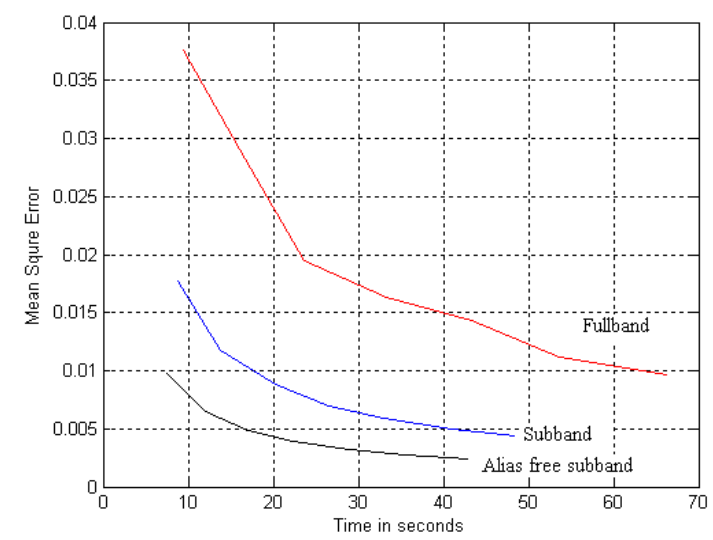

Fig. 12.Convergence Comparison between Full Band, Subband and Alias Free Subband Convergence

Simulations results show that the Alias free subband adaptive filter structure achieves better convergence rate than the full band structure for random noise input at lower computational complexity

Table 1: Error and Convergence Comparision

\begin{tabular}{|c|c|c|c|c|c|c|}
\hline \multirow{2}{*}{$\begin{array}{l}\text { No. of } \\
\text { Iterations }\end{array}$} & \multicolumn{2}{|c|}{ Full band } & \multicolumn{2}{c|}{ Sub band } & \multicolumn{2}{|c|}{ Alias free Sub band } \\
\cline { 2 - 7 } & Error & $\begin{array}{l}\text { Time in } \\
\text { Sec }\end{array}$ & Error & $\begin{array}{l}\text { Time in } \\
\text { Sec }\end{array}$ & Error & $\begin{array}{l}\text { Time in } \\
\text { Sec }\end{array}$ \\
\hline 100 & 0.0376 & 9.384 & 0.0177 & 8.772 & 0.0098 & 7.491 \\
\hline 150 & 0.0291 & 15.983 & 0.0118 & 13.86 & 0.0065 & 11.998 \\
\hline 200 & 0.0195 & 23.604 & 0.0088 & 20.179 & 0.0048 & 17.004 \\
\hline 250 & 0.0163 & 33.238 & 0.0070 & 26.538 & 0.0039 & 22.272 \\
\hline 300 & 0.0143 & 43.342 & 0.0059 & 32.918 & 0.0032 & 28.932 \\
\hline 350 & 0.0112 & 53.657 & 0.0050 & 40.998 & 0.0028 & 35.491 \\
\hline 400 & 0.0096 & 66.425 & 0.0044 & 48.47 & 0.0024 & 42.952 \\
\hline
\end{tabular}

In this table observe that while comparing fullband, subband adaptive filter and Alias free subband adaptive filter, the Alias free subband adaptive filter error is very less than subband adaptive filter, fullband adaptive filter. Also comparing fullband , subband adaptive filter and Alias free subband adaptive filter, the Alias free subband adaptive filter convergence is very fast than fullband adaptive filter, subband adaptive filter . Because of in which the input is decomposed into a number of subband signals, and the adaptive filtering is performed on each subband. The computational complexity of the proposed SAF algorithm is approximately reduced compared to that of the full band algorithm. Simulations results show that the proposed sub band structure achieves better convergence rate than the full band structure for random noise input at lower computational complexity

\section{CONCLUSION}

In this Paper, in order to fully exploit the benefits of SAF, a structure with critical sampling that is virtually free Interband aliasing is proposed. The inter band aliasing is extracted in each sub band using the bandwidth-increased FIR linear-phase analysis filters and then subtracted from each sub band signal. The use of the bandwidth-increased analysis filters introduces an extra computational load. The almost alias-free sub band signals have spectral dips, so the spectral dips are reduced using a filter for the spectral flatness and then the outputs are used for adaptive filtering in each sub band. The computational complexity of the proposed SAF algorithm is approximately reduced compared to that of the full band algorithm. Simulations results show that the proposed sub band structure achieves better convergence rate than the full band structure for random noise input at lower computational complexity.

\section{ACKNOWLEDGMENTS}

The Authors wish to thank Dr.Kota.Srinivas, D.Tirumala Rao, V.JaganNaveen,Ch.V.A.Priyadarshini, A.L.Prasanthi ,G.Vijaya Santhi and U.Naresh Kumar for their sugessions which have the improved the presentation of the material in this paper.

\section{REFERENCES}

[1] Adaptive Filter Theory by Simon Haykin,Pearson.

[2] Discrete-Time Signal Processing by A. V. Oppenheim and R. W. Schafer,PHI.

[3] Digital Signal Processing: Principles, Algorithms, and Applications by J. G. Proakis and D. G. Manolakis,PHI

[4] MATLAB7-Rudra Pratap, Oxford University Press.

[5] B. Widrow and S. D. Stearns, Adaptive Signal Processing, Second Edition, Pearson.

[6] Digital Signal Processing- Sanjit K.Mitra, Second Edition.

[7] MATLAB Programming- Y.Kirani Singh,B.B.Chaudhuri.

[8] SangGyun Kim, Chang D. Yoo, and Truong Q. Nguyen "Alias-Free Subband Adaptive Filtering With Critical Sampling" IEEE Transactions On Signal Processing, Vol. 56, No. 5, May 2008,pp.1894-1904.

[9] SangGyun Kim, Chang D. Yoo, and Truong Q. Nguyen "Alias-Free Subband Adaptive Filtering With Critical Sampling” IEEE Transactions On Signal Processing, Vol. 56, No. 5, May 2008,pp.1894-1904.

[10] Christian Schüldt,Fredric Lindstrom, and Ingvar Claesson "A Low-Complexity Delayless Selective Sub band Adaptive Filtering Algorithm" IEEE Transactions On Signal Processing, Vol. 56, No. 12, December 2008

T.Prabhakar received B.E Electronics and Communication Engineering from Andhra University and M.Tech Instrumentation and Control from JNTU Kakinada. He is working as Asst. Professor in GMR Institute of Technology, Rajam since 2002. His research interests are in Signal Processing, Communication and Image Processing.

Dr. K. Satya Prasad has more than 31 years of Experience in teaching and 20 years of $\mathrm{R} \& \mathrm{D}$. He is an expert in Digital Signal Processing. He produced $4 \mathrm{PhD}$ 's and guiding $10 \mathrm{PhD}$ scholars. He authored Electronic Devices and Circuits text book. $\mathrm{He}$ held different positions in his carrier like Head of the Department, Vice Principal, and Principal for JNTU Engineering College. He published more than 30 technical papers in national and International journals and conferences.

P.M.K.Prasad Graduated from IETE, New Delhi and M.E in Systems and Signal Processing from O.U Hyderabad. He is working as Associate Professor in GMR Institute of Technology, Rajam since 2006. His research interests are in Signal Processing, Communication and Image Processing. 